\title{
Diabetic Amyotrophy: From the Basics to the Bedside
}
Authors:
*James W. Albers, ${ }^{1}$ Ryan D. Jacobson,, ${ }^{2}$ David L. Smyth ${ }^{2}$
1. University of Michigan Medical School, Ann Arbor, Michigan, USA
2. Department of Neurology, Rush University Medical Center, Chicago, Illinois, USA
*Correspondence to jwalbers@umich.edu
Disclosure: $\quad$ The authors have declared no conflicts of interest.
Received:
06.09.19
Accepted:
12.11.19
Keywords:
Bruns-Garland syndrome, diabetic amyotrophy, diabetic lumbosacral radiculoplexus neuropathy (DLRPN).
Citation:
EMJ. 2020;5[1]:94-103.

\begin{abstract}
Diabetic amyotrophy is a rare complication of diabetes compared to distal symmetric polyneuropathy, but can occasionally be encountered in clinical practice, particularly as the incidence of diabetes increases. The distinctive history of unilateral neuropathic symptoms followed rapidly by atrophy and weakness is typical of the disorder. This complication most commonly occurs in cases of well-controlled Type 2 diabetes mellitus. While the underlying pathophysiology is known to be microvasculitic in nature, the diagnosis is often based on clinical and electrodiagnostic grounds and tissue biopsy is not typically performed. Attempts at corticosteroid administration during immunotherapy should be carefully considered on a patient-by-patient basis. Better recognition of this disorder is likely to result in more rapid diagnosis, counselling, and subspecialty referral.
\end{abstract}

\section{INTRODUCTION}

Among the most common and costly complications of diabetes is peripheral neuropathy. Most often, this takes the form of a distal symmetric polyneuropathy, manifesting as a length-dependent symmetric neuropathy characterised primarily by distal sensory and motor symptoms. It is important, however, for clinicians to be aware of other phenotypes of neuropathy that may occur in the setting of diabetes. One neuropathic condition of key importance is diabetic amyotrophy, which the authors review in this article.

Diabetic amyotrophy is a subacute, progressive, and often unilateral neurogenic process that occurs in the setting of diabetes. The typical

course is one of severe pain, followed soon thereafter by weakness and wasting with weight loss. This condition has been reported in the literature over many decades. An early term for this syndrome was 'Bruns-Garland syndrome'. Bruns described the syndrome in 1890, ${ }^{1}$ and in 1955, Garland published a case-series of nine patients whom he assessed to have diabetic amyotrophy. ${ }^{2}$ Garland, an English neurologist, astutely observed that the syndrome was characterised by asymmetric, lower extremity symptoms occurring in patients with diabetes who typically had a short and "not severe" course of diabetes.

The terminology surrounding this condition is varied, which may add confusion. More often, 'Bruns-Garland' syndrome is now referred to 
as diabetic amyotrophy. Other terms exist in practice and the literature, including femoral neuropathy and proximal motor neuropathy. Diabetic lumbosacral radiculoplexus neuropathy (DLRPN) is a term commonly used and may be useful in drawing similarity to diabetic cervical radiculoplexus neuropathy, a similar syndrome affecting the upper extremities.

\section{METHODS}

The data included in this review was summarised from articles identified on MEDLINE, PubMed, and Google Scholar searches in July 2019, in which citations involving "diabetic amyotrophy", "diabetic lumbosacral radiculoplexus neuropathy", "Bruns-Garland syndrome", or "proximal diabetic neuropathy" were found. The titles and abstracts of the resulting articles were screened to select those with relevance. Namely, we included all articles that appear to describe clinical features of patients diagnosed with diabetic amyotrophy or any of the related terms associated with this condition. The bibliographies of the obtained articles were reviewed to identify additional articles. The search was restricted by language to the extent that English abstracts were required. Individual cases that included information about the symptoms, signs, evaluation, treatment, and course were included. The PRISMA checklist and guidelines were followed, to the extent that they were relevant to the review of observational studies. The review was not designed to capture all the relevant articles but to be highly representative of the existing literature involving diabetic amyotrophy. The search resulted in selection of 20 case report articles ${ }^{3-22}$ and 8 observational case-series, ${ }^{2,23-29}$ from which data was available for a total of 115 cases.

\section{CLINICAL FEATURES}

The characteristics of 115 cases of diabetic amyotrophy, 95\% of whom had Type 2 diabetes mellitus, are summarised in Table 1. The median age was 62 years, the duration of diabetes preceding the onset of diabetic amyotrophy was relatively short (median duration of 3 years), and nearly one-fifth of cases were diagnosed with diabetes during the evaluation of amyotrophy. Overall, the diabetes was well-controlled and nonsevere; although approximately one-third were being treated with insulin. There were few known diabetic complications (neuropathy, retinopathy, or nephropathy) consistent with the relatively brief duration of diabetes. Weight loss beginning coincidentally with the onset of symptoms was common and occurred in up to two-thirds of cases, ranging from 4.5 to $54.5 \mathrm{~kg}$.

Cardinal symptoms included the abrupt onset of severe proximal leg pain involving the thigh, hip, or back, followed by progressive weakness and atrophy within weeks. Pain was unilateral in $70 \%$ of cases; when bilateral, it was usually asymmetric. A minority of cases complained of symmetric back and leg pain. The pain itself was characterised as deep and aching as well as tingling with or without burning. Proximal or proximal>distal weakness was the most characteristic sign, accompanied by variable amounts of muscle atrophy. The distribution of weakness and atrophy was unilateral in $25 \%$ of cases, and bilateral but asymmetric in $>50 \%$ of cases. Overall, involvement of the other leg occurred in approximately half of the cases, typically within 6 months. A small number of cases that had an otherwise typical presentation of diabetic amyotrophy then developed diffuse weakness progressing to severe quadriparesis. Sensory loss was common but not particularly useful for diagnostic purposes because examination usually revealed symmetric distal sensory loss. In up to $20 \%$ of cases there was evidence of dysautonomia, including orthostatic hypotension. The most common reflex abnormalities included absent knee and ankle reflexes (33\%) or asymmetric knee reflexes (27\%), and $6 \%$ of cases were known to have a polyneuropathy prior to the onset of the diabetic amyotrophy. Nevertheless, $>50 \%$ of cases had clinical evidence of an underlying diabetic polyneuropathy at initial evaluation.

These results obtained from 115 cases reported individually in the literature are supported by the overall results from several important observational case-series reports. For example, Barohn et al. ${ }^{23}$ described 17 patients with diabetic amyotrophy, all of whom had a preceding diagnosis of diabetes (three controlled with insulin, six with oral antihyperglycaemics, and three with diet alone) or were diagnosed at time of presentation. 
Table 1: Characteristics of 115 cases of diabetic amyotrophy at time of initial diagnosis and follow-up evaluations.

\begin{tabular}{|c|c|c|c|c|c|c|}
\hline Characteristics & $\begin{array}{l}\text { Number of } \\
\text { cases }(n)\end{array}$ & Yes (\%) & Mean & Median & Range & $\begin{array}{l}5-95^{\text {th }} \\
\text { percentile }\end{array}$ \\
\hline Age (years) & 115 & & 61.0 & 62.0 & $13.0-80.0$ & $43.0-76.0$ \\
\hline Sex (male) & 115 & $76(66)$ & & & & \\
\hline T1DM & 115 & $6(5)$ & & & & \\
\hline T2DM & 115 & $109(95)$ & & & & \\
\hline Diabetes known pre-DA & 115 & $94(82)$ & & & & \\
\hline Diabetes duration (years) & 86 & & 6.0 & 3.0 & $0.1-30.0$ & $0.1-20.0$ \\
\hline Dx $\leq 3$ months, pre-DA & 13 & $13(11)$ & & & & \\
\hline Dx made during evaluation & 115 & $21(18)$ & & & & \\
\hline \multicolumn{7}{|l|}{ Diabetes treatment (pre-DA) } \\
\hline Insulin & 115 & $27(34)$ & & & & \\
\hline Duration (months) & 9 & & 39.0 & 36.0 & $0.5-120.0$ & $1.0-96.0$ \\
\hline $\mathrm{OHG}$ & 115 & $22(28)$ & & & & \\
\hline Duration (months) & 12 & & 59.0 & 36.0 & $0.5-180.0$ & $1-180.0$ \\
\hline Insulin and OHG & 115 & $4(4)$ & & & & \\
\hline Duration (months) & 3 & & 20.0 & 24.0 & $0.5-36.0$ & $3.0-35.0$ \\
\hline Diet only or no treatment & 115 & $15(13)$ & & & & \\
\hline Unknown but not insulin & 115 & $21(18)$ & & & & \\
\hline Unknown & 115 & $26(23)$ & & & & \\
\hline Start insulin $\leq 2$ months pre-DA & 89 & $2(2)$ & & & & \\
\hline Associated features & 115 & & & & & \\
\hline Weight loss preceding DA & 115 & $6(5)$ & & & & \\
\hline Amount (kg) & 6 & & 16.8 & 14.5 & $6.8-31.8$ & $8.6-28.6$ \\
\hline Interval (months) & 6 & & 5.0 & 4.0 & $2.0-12.0$ & $2.0-11.0$ \\
\hline Weight loss coincident DA & 115 & $72(63)$ & & & & \\
\hline Amount (kg) & 72 & & 11.4 & 11.8 & $4.5-54.5$ & $4.5-18.2$ \\
\hline $\begin{array}{l}\text { Known diabetic DSPN } \\
\text { (polyneuropathy) }\end{array}$ & 115 & $7(6)$ & & & & \\
\hline Clinically evident DSPN & 113 & $58(51)$ & & & & \\
\hline Diabetic retinopathy & 115 & $10(9)$ & & & & \\
\hline Diabetic nephropathy & 115 & $4(3)$ & & & & \\
\hline Illness/surgery & 115 & 0 & & & & \\
\hline Duration of symptoms at Dx (months) & 98 & & 6.0 & 4.0 & $0.3-24.0$ & $\mid 1.0-12.0$ \\
\hline \multicolumn{7}{|l|}{ Symptoms } \\
\hline Pain only & 115 & $4(3)$ & & & & \\
\hline Weakness only & 115 & $11(10)$ & & & & \\
\hline Pain followed by weakness & 115 & $87(76)$ & & & & \\
\hline Intervening interval (weeks) & 41 & & 2.0 & 2.0 & $0.0-10.0$ & $0.0-8.0$ \\
\hline Weakness followed by pain & 115 & $5(4)$ & & & & \\
\hline Pain and weakness coincident & 115 & $8(7)$ & & & & \\
\hline Autonomic involvement & 115 & $21(18)$ & & & & \\
\hline \multicolumn{7}{|l|}{ Pain distribution } \\
\hline Unilateral & 101 & $71(70)$ & & & & \\
\hline Proximal predilection & 71 & $66(93)$ & & & & \\
\hline Back and leg & 71 & $4(6)$ & & & & \\
\hline
\end{tabular}


Table 1 continued.

\begin{tabular}{|c|c|c|c|c|c|c|}
\hline Characteristics & $\begin{array}{l}\text { Number of } \\
\text { cases }(n)\end{array}$ & Yes (\%) & Mean & Median & Range & $\begin{array}{l}5-95^{\text {th }} \\
\text { percentile }\end{array}$ \\
\hline Distal & 71 & $1(1)$ & & & & \\
\hline Asymmetric & 101 & $13(13)$ & & & & \\
\hline Proximal predilection & 13 & $13(100)$ & & & & \\
\hline Symmetric & 101 & $17(17)$ & & & & \\
\hline Proximal predilection & 17 & $6(35)$ & & & & \\
\hline Back and legs or diffuse legs & 17 & $10(59)$ & & & & \\
\hline Distal legs & 17 & $1(6)$ & & & & \\
\hline \multicolumn{7}{|l|}{ Signs } \\
\hline Sensory impairment and distribution & 74 & $54(68)$ & & & & \\
\hline Unilateral & 55 & $14(25)$ & & & & \\
\hline Asymmetric & 55 & $9(16)$ & & & & \\
\hline Symmetric (distal symmetric) & 55 & $32(58)$ & & & & \\
\hline Weakness/atrophy distribution & 115 & $110(96)$ & & & & \\
\hline Unilateral & 110 & $28(25)$ & & & & \\
\hline Asymmetric & 110 & $59(54)$ & & & & \\
\hline Symmetric & 110 & $23(21)$ & & & & \\
\hline Weakest muscle MRC grade & 49 & & 2.3 & 2.0 & $0.0-0.5$ & $0.0-0.4$ \\
\hline \multicolumn{7}{|l|}{ Weakness/atrophy pattern } \\
\hline Proximal & 110 & $58(53)$ & & & & \\
\hline Proximal>distal & 110 & $37(34)$ & & & & \\
\hline Distal & 110 & $15(14)$ & & & & \\
\hline Abnormal reflexes & 78 & $78(100)$ & & & & \\
\hline Asymmetric knees & 78 & $21(27)$ & & & & \\
\hline Asymmetric knees and ankles & 78 & $4(5)$ & & & & \\
\hline Asymmetric knees, absent ankles & 78 & $11(14)$ & & & & \\
\hline Absent knees & 78 & $8(10)$ & & & & \\
\hline Absent knee/asymmetric ankles & 78 & $1(1)$ & & & & \\
\hline Absent knees and ankles & 78 & $26(33)$ & & & & \\
\hline Asymmetric ankles & 78 & $3(4)$ & & & & \\
\hline Absent ankles & 78 & $4(5)$ & & & & \\
\hline Clinically evident underlying DSPN & 113 & $58(51)$ & & & & \\
\hline \multicolumn{7}{|l|}{ Laboratory results } \\
\hline HbA1c (\%) & 65 & & 8.5 & 8.0 & 5.8-19.8 & $6.0-12.0$ \\
\hline Fasting blood glucose (mg\%) & 33 & & 200.0 & 170.0 & $81.0-414.0$ & $118.0-356.0$ \\
\hline Elevated ESR (no [\%], mm/hour) & 48 & $20(42)$ & 19.0 & 8.0 & $0.0-78.0$ & $5.0-36.0$ \\
\hline Elevated CSF protein (no [\%], mg\%) & 64 & $56(88)$ & 117.0 & 90.0 & $\begin{array}{l}30.0- \\
1,560.0 \\
\end{array}$ & $43.0-212.0$ \\
\hline Abnormal needle EMG (denervation) & 40 & $37(93)$ & & & & \\
\hline $\begin{array}{l}\text { Asymmetric } \\
\text { proximal>distal }\end{array}$ & 40 & $25(63)$ & & & & \\
\hline Symmetric proximal>distal & 40 & $12(30)$ & & & & \\
\hline Paraspinal muscles involved & 37 & $36(97)$ & & & & \\
\hline EMG evidence of diabetic DSPN & 34 & $31(91)$ & & & & \\
\hline \multicolumn{7}{|l|}{ Treatment } \\
\hline
\end{tabular}


Table 1 continued.

\begin{tabular}{|c|c|c|c|c|c|c|}
\hline Characteristics & $\begin{array}{l}\text { Number of } \\
\text { cases }(n)\end{array}$ & Yes (\%) & Mean & Median & Range & $\begin{array}{l}5-95^{\text {th }} \\
\text { percentile }\end{array}$ \\
\hline $\begin{array}{l}\text { Intravenous Ig, Solu-medrol }{ }^{\oplus} \text {, } \\
\text { corticosteroids }\end{array}$ & $97^{*}$ & $16(16)$ & & & & \\
\hline Improved glycaemic control & 97 & $24(25)$ & & & & \\
\hline Started insulin & 97 & $13(13)$ & & & & \\
\hline Opioid analgesics & 97 & $28(76)$ & & & & \\
\hline None aside from symptomatic & 97 & $26(26)$ & & & & \\
\hline \multicolumn{7}{|l|}{ Outcome } \\
\hline Death & 96 & $2(2)^{* *}$ & & & & \\
\hline Progression/no/minimal improvement & 96 & $3(3)$ & & & & \\
\hline Partial improvement (wheelchair) & 96 & $4(4)$ & & & & \\
\hline Moderate improvement (cane) & 96 & $40(42)$ & & & & \\
\hline Substantial improvement (no aids) & 96 & $35(36)$ & & & & \\
\hline Full recovery & 96 & $12(13)$ & & & & \\
\hline Time to nadir after Dx (months) & 32 & & 5.0 & 4.0 & $0.5-14.0$ & $1.0-12.0$ \\
\hline Residual symptoms & 65 & $56(86)$ & & & & \\
\hline Weakness/atrophy & 56 & $46(82)$ & & & & \\
\hline $\begin{array}{l}\text { Pain (although most had } \\
\text { improved) }\end{array}$ & 56 & $19(34)$ & & & & \\
\hline $\begin{array}{l}\text { Contralateral leg involvement (any } \\
\text { time) }\end{array}$ & 71 & $46(64)$ & & & & \\
\hline Arm involvement (any time) & 115 & $21(18)$ & & & & \\
\hline Thoracic radiculopathy (any time) & 115 & $15(13)$ & & & & \\
\hline Interval (months) & 71 & & 5.0 & 3.0 & $0.0-60.0$ & $0.0-11.0$ \\
\hline $\begin{array}{l}\text { Time of re-evaluation after onset } \\
\text { (months) }\end{array}$ & 74 & & 17.0 & 12.0 & $0.8-42.0$ & $3.0-36.0$ \\
\hline
\end{tabular}

CSF: cerebrospinal fluid; DA: diabetic amyotrophy; DSPN: distal symmetric peripheral neuropathy; Dx: diagnosis; EMG: electromyography; ESR: erythrocyte sedimentation rate; IV: intravenous; MRC: Medical Research Council; OHG: oral hypoglycaemic; T1DM: Type 1 diabetes mellitus; T2DM: Type 2 diabetes mellitus.

*3 cases treated with intravenous Ig and improved glycaemic control.

${ }^{* *} 1$ case developed acute tubular necrosis and aspiration pneumonia after treatment with intravenous Ig, intravenous cyclophosphamide, and methylprednisolone.

Of the cohort, 14 reported unilateral and three reported bilateral pain at onset, though at time of evaluation pain had become bilateral in all. Latency to involvement of the other leg ranged from 3 days to 8 months. A minority of patients complained of lower extremity paresthesias or numbness, though examination often revealed distal sensory loss consistent with an underlying polyneuropathy. Five patients experienced weight loss, ranging from 13.5 to $36.0 \mathrm{~kg}$. Similarly, Dyck et al. ${ }^{25}$ reported 33 patients with diabetic amyotrophy with similarly identified risk factors and initial symptoms.

The median age at onset was 65.0 years with a median duration of diabetes of 4.1 years; all but one had Type 2 diabetes mellitus. Pain was the most common or severe symptom at onset in 27 patients in this group; however, weakness became the more bothersome symptom later in the course of the disease. Progression to bilateral involvement occurred with a median of 3 months and median weight loss associated with the disease was $13.6 \mathrm{~kg}$. 
The differential diagnosis for a lumbosacral plexopathy with similar presenting symptoms and signs includes inflammatory (e.g., herpes zoster), ischaemic (e.g., postradiation), and mechanical compression, including neoplastic involvement of the plexus. Additional evaluation may be performed in patients with a known or suspected malignancy. Non-DLRPN has been described as an inherently identical syndrome, also of microvasculitic origin, affecting nondiabetic individuals.

\section{PATHOPHYSIOLOGY}

The subacute time course and painful nature of diabetic amyotrophy are suggestive of a process with vascular or ischaemic causes. This has played out in pathologic studies of DLRPN. Said et al. ${ }^{29}$ published their study of 10 diabetic patients with painful, proximal, asymmetric neuropathies who underwent biopsy of the intermediate cutaneous nerve of the thigh. Three biopsies showed apparent changes of ischaemia, including an inflammatory infiltrate or vasculitis in two of the biopsies. In 1999, Dyck et al. ${ }^{25}$ published their case-series of 33 patients with diabetic amyotrophy who underwent biopsy of either the sural or superficial peroneal nerve. ${ }^{25}$ Of these 33 nerve biopsies, many showed changes indicative of vasculopathy, including focal or multifocal nerve fibre degeneration in 19 and epineurial neovascularisation in 21. Inflammatory changes were found in all nerves, and at a rate significantly more frequent than in patients with typical diabetic polyneuropathy or control nerves. Small arterioles, venules, and capillaries were most frequently involved. Two nerves showed a necrotising vasculitis while 13 others showed changes at least suggestive of a necrotising vasculitis. Similar pathologic changes of ischaemia and microvasculitis have been observed in non-DLRPN, a similar clinical entity in patients without a diagnosis of diabetes. ${ }^{30}$ Subsequently, immunostaining has been carried out on nerve biopsies of patients with either diabetic or nondiabetic RPN. These studies have revealed increased ICAM-1 positive cells in blood vessels and increased NF-kB staining in blood vessels. Both findings support an underlying dysimmune vascular basis for these disorders. ${ }^{31}$
These observations pathophysiology of diabetic amyotrophy have also been supported by additional studies. In 1998, Llewelyn et al. ${ }^{28}$ published a case-series of 14 patients who underwent nerve biopsy in the evaluation of proximal diabetic neuropathy. ${ }^{28}$ In this cohort, biopsies of the intermediate cutaneous nerve of the thigh were performed. Three out of 14 patients showed inflammation in epineurial vessels, and more mild changes were observed in a fourth. They also included one sural nerve biopsy specimen in their series, which demonstrated inflammatory mononuclear infiltration in an arteriole. Younger et al. ${ }^{22}$ published a post-mortem analysis of autopsy data on a 59-year-old man with apparent diabetic amyotrophy who died following treatment with intravenous Ig and cyclophosphamide. ${ }^{22}$ Femoral nerve tissue and lumbar plexus tissue showed perivascular epineurial inflammation. Nerve biopsy is not routinely required or performed in the workup and evaluation of a patient with suspected diabetic amyotrophy.

\section{CLINICAL EVALUATION}

There is no single test that is diagnostic of diabetic amyotrophy. The evaluation should begin with a thorough history check and comprehensive neurological examination. The history typically discloses an acute to subacute, progressive time course. Frequent examination features include atrophy and loss of reflexes in the affected limb or limbs along with motor weakness. Given the presence of diabetes, an underlying distal symmetric polyneuropathy is common. Red flag features that may prompt a broader work-up include a lack of sensory symptoms or the presence of upper motor neuron signs, both of which may imply an alternative disease process including worrisome possibilities such as amyotrophic lateral sclerosis. Involvement beyond the lower extremities is also nontypical in DLRPN and would likely prompt additional evaluation.

Table 1 summarises the application and utility of different diagnostic tests in the evaluation of suspected diabetic amyotrophy. The Table does not include imaging of the lumbosacral spine, although $\mathrm{MRI}$ is usually performed to exclude an unsuspected structural explanation and 
electromyography (EMG) is also a beneficial test in this setting. The expected findings include active denervation in clinically affected muscles, typically in a pattern suggestive of a lumbar plexopathy. As diabetic amyotrophy is truly a radiculoplexus neuropathy, involvement of the paraspinal muscles is common due to injury at the level of the nerve root and the nerve conduction studies may be impacted by the presence of underlying polyneuropathy. In this current literature review, 37 of 40 patients had abnormal needle EMG studies. The electrodiagnostic findings were asymmetric approximately two-thirds of the time. Of 37 patients, 31 had evidence of underlying diabetic polyneuropathy. EMG features that should prompt consideration of alternative diagnoses include a normal study or a lack of ongoing denervation, neither of which is typical.

Cerebrospinal fluid (CSF) analysis may be considered. The typical finding in diabetic amyotrophy is that of elevated protein. In this review, 56 of 64 patients with available CSF studies had an elevated protein level (Table 1), the median being $90 \mathrm{mg} / \mathrm{dL}$. The presence of a significant pleocytosis is atypical and may prompt additional consideration of mimics of diabetic amyotrophy. It is not the authors' general practice to perform a lumbar puncture on all patients with suspected diabetic amyotrophy. This test is invasive, and an elevated CSF protein is a nonspecific finding, especially in the setting of diabetes in which elevated CSF protein levels are frequently incidental.

With a supportive history and examination findings, the yield of any blood test is limited. If the prior probability of diabetic amyotrophy is high, then serum work-up for other autoimmune conditions, including connective tissue diseases or vasculitides, is unlikely to be impactful. In this review, the sedimentation rate was elevated in 20 of 48 patients in whom it was measured. As the sedimentation rate itself is nonspecific, it should be interpreted with caution. Because diabetic amyotrophy is a complication of underlying diabetes it often prompts some reevaluation of the patient's underlying diabetic control. This is classically indicated in patients with well-controlled diabetes. In 65 patients reviewed with available $\mathrm{HbA1c}$ values, the median level was 8.0\% (5.8-19.8\%) (Table 1).
In summary, the evaluation of diabetic amyotrophy is typically driven by clinical suspicion, supported by a suggestive history and typical examination findings. EMG testing is a common and reasonable element of this evaluation to support the clinical impression. Other studies, including CSF and blood tests, are unlikely to offer additional insight. Nerve biopsy is usually not needed to reach this diagnosis.

\section{TREATMENT}

The treatment of diabetic amyotrophy remains somewhat controversial with a lack of high-quality evidence from well-designed clinical trials. Given the evidence supporting an underlying microvasculitic basis for the disease process, immunotherapy is certainly of interest. Before approaching this difficult question, clinicians may consider basic supportive measures in the treatment and management of these patients. Although diabetic amyotrophy most often occurs in patients with wellcontrolled diabetes, presentation with this syndrome merits re-examination of the patient's glycaemic control. Physical therapy is warranted to enhance a patient's mobility. Assistive devices or bracing may be needed depending upon an individual's degree and distribution of weakness.

Corticosteroids are often employed in the treatment of patients with diabetic amyotrophy. A 2006 study by Dyck et al. ${ }^{32}$ is often cited but only published in abstract form. In this study, 75 patients were studied, of whom 49 received corticosteroids. The primary endpoint of motor improvement was not significantly different in those who received treatment; however, secondary endpoints, such as neuropathic symptoms, were better in the treatment group.

A 1995 study by Krendel et al. ${ }^{33}$ reviewed the response to treatment of 15 patients with proximal, axonal diabetic neuropathies. Twelve of these patients received intravenous ig showing benefits in strength. Most of these patients also received steroids orally or intravenously. Two patients in these series received intravenous cyclophosphamide, and one was started on azathioprine as a steroidsparing agent. Pascoe et al. ${ }^{34}$ published their series of 12 patients, which included five who 
underwent plasma exchange, three who received intravenous Ig, and one who received both treatments. ${ }^{34}$ Most patients improved, including four of the five plasma exchange patients and two of the three intravenous Ig treatment patients. Jaradeh et al. ${ }^{35}$ studied 15 patients with diabetes and diabetic amyotrophy, including nine who received plasma exchange and six who received intravenous $1 \mathrm{~g}^{35}$ All exhibited improvements in their pain and neuropathy disability scores.

While these data are exciting and plausible in the setting of the known microvasculitic aetiology for diabetic amyotrophy, some clinicians may be cautious. None of the last three case-series were randomised controlled trials. Further, diabetic amyotrophy is a condition with a natural history that tends towards improvement and resolution of symptoms, making the lack of a control group especially conspicuous. Intravenous Ig and plasma exchange, while frequently used for autoimmune neuromuscular conditions, are invasive and not without potential complications. A recently updated Cochrane review concluded there is currently no evidence from randomised trials to support a positive or negative effect of any immunotherapy in the treatment in diabetic amyotrophy. ${ }^{36}$ Steroids are considered, particularly in cases of relatively recent onset, with progressing weakness or with especially severe neuropathic pain.

Although immune-mediated treatments may improve pain during the acute phase, pain is typically treated with agents shown to be effective for painful diabetic neuropathy, including tricyclic antidepressants, the selective serotonin and noradrenaline reuptake inhibitors, anticonvulsants, membrane stabilisers, topical agents such as capsaicin, and opioids. ${ }^{37}$ When pain is particularly severe, hospitalisation may be required to achieve pain control. ${ }^{38}$

\section{PROGNOSIS}

Because diabetic amyotrophy is a painful condition accompanied by significant motor weakness, patients are likely to present to their primary physician, endocrinologist, or a neurologist early in the disease course or while their motor weakness is most disabling. After the initial presentation, progression over months to involve the opposite leg, a thoracic nerve root, or even the arm is not uncommon. Nonetheless, the prognosis for eventual improvement in both sensory and motor symptoms is good, and multiple studies have demonstrated that cessation of pain and recovery of normal or near-normal strength are typical.

In 1972, Coppack et al. ${ }^{39}$ followed a group of 27 patients for a mean of 61 months. Most patients had excellent recovery at 18 months. Pain recovered first, typically within 1 year, followed by improved strength and, to a lesser extent, recovery of the knee reflex. None of the patients had persistent disabilities, although persistent atrophy or hyporeflexia was common. Also in 1972, Casey and Harrison ${ }^{24}$ published their series of 12 patients who were followed for a mean of 12 years. Only two patients had persistent pain, which was mild. A single patient did not report functional improvement, and the examiners also reported that muscle strength had significantly improved in all but one patient. Interestingly, many patients in this study were newly diagnosed with diabetes at the time of presentation. Improvement in the neuromuscular problem was noted to coincide with the initiation of oral medications or improved glycaemic control.

Even in cases with proven vasculitis on tissue pathology, the prognosis is very good. Said ${ }^{29}$ reported on four patients with nerve biopsies demonstrating the typical inflammatory or vasculitic features of diabetic amyotrophy. In all four, pain was very severe but remitted soon after the nerve biopsy was performed. Two of the four had complete motor recovery, while the two others had mild residual weakness, which was attributed to a lumbar herniated disc in one case.

Dyck et al. ${ }^{40}$ also studied 33 patients with diabetic amyotrophy, for the purpose of comparison to the more seldom studied nondiabetic radiculoplexus neuropathy. Of the diabetic patients, 13 reported severe pain at the time of initial evaluation compared to six at the time of a telephone follow-up. The number of patients reporting significant weakness did not change between the time of initial evaluation and telephone follow-up. While this may imply a somewhat poorer prognosis for motor recovery, it is worth noting that the number of 
patients requiring a wheelchair for ambulation declined from 16 to three. Furthermore, the median time to telephone follow-up in these diabetic patients was 25.9 months, less than some of the other studies included in this review.

Diabetic amyotrophy is a painful condition that can be acutely disabling. Even in the absence of immunotherapies, the prognosis for the improvement of both sensory symptoms and for motor recovery is good. Patients are often devastated and can be depressed by the rapid onset of pain and weakness, making it important that they are reassured using guarded optimism that there will be improvement. This favourable natural history should factor into decision making regarding any proposed trial of immunotherapy.

\section{CONCLUSION AND FUTURE DIRECTIONS}

While diabetic amyotrophy is a rare complication of diabetes, it is encountered frequently enough in clinical practice that non-neurologists and neurologists alike are likely to encounter it, especially as the incidence of diabetes increases. It is important for clinicians to carefully consider the clinical history, because the distinctive timeline of unilateral or asymmetric neuropathic symptoms followed by weakness and atrophy is distinct from the typical story of diabetic polyneuropathy. Importantly, this complication most commonly occurs in patients with well-controlled Type 2 diabetes mellitus and may even be the presenting feature of diabetes. While the underlying pathophysiology is known to be microvasculitic in nature, immunotherapy is still controversial. The use of corticosteroids in particular must be carefully weighed in each case. Better recognition of this disorder is likely to result in more rapid diagnosis, counselling, and subspecialty referral. Improved understanding of the underlying pathophysiology of this heterogenous syndrome will direct future treatment protocols.

\section{References}

1. Bruns L. Ueber neuritische Lahmungen beim diabetes mellitus. Berl Klin Wochenschr. 1890;27:509-1. (In German).

2. Garland H. Diabetic amyotrophy. $\mathrm{Br}$ Med J. 1955;26(2):1287-90.

3. Bokhari SRA et al. The Syndrome of diabetic amyotrophy: a preventable disaster? Journal Coll Physicians Surg Pak. 2018;28(6):S91-3.

4. Chokroverty S, Sander HW. AAEM case report \#13: diabetic amyotrophy. Muscle Nerve. 1996;19(8):939-45.

5. Courtney AE et al. Human immunoglobulin for diabetic amyotrophy--a promising prospect? Postgrad Med J. 2001;77(907):326-8.

6. Davidson RA et al. Bruns-Garland syndrome (diabetic amyotrophy). Orthopedics. 2003;26(1):87-8.

7. Engel PA, Qian J. Diabetic amyotrophy and autoimmune thyroiditis: are they related? J Am Geriatr Soc. 2004;52(8):1408-9.

8. Fernandes Filho JA et al. Diabetic amyotrophy in an adolescent responsive to intravenous immunoglobulin. Muscle Nerve. 2005;32(6):818-20.

9. Flatow EL, Michelsen CB. Diabetic amyotrophy. A case report. J Bone
Joint Surg Am. 1985;67(7):1132-5.

10. Greenberg JS et al. Evaluation and rehabilitation of a patient with diabetic lumbosacral radiculoplexus neuropathy. PM \& R. 2009;1(8):774-7.

11. Jones KK, Walsh JR. Diabetic amyotrophy. Postgrad Med 1965;37(3):342-7.

12. Koca TT. Concomitance of diabetic neuropathic amyotrophy and cachexia: a case report with review of the literature. North Clin Istanb. 2015;2(2):165-70

13. Massey EW. Diabetic neuropathic cachexia and diabetic amyotrophy. Acta Diabetol Lat. 1982;19(1):91-5.

14. McCormack EP et al. Use of MRI in diabetic lumbosacral radiculoplexus neuropathy: case report and review of the literature. Acta Neurochir (Wien). 2018;160(11):2225-7.

15. Ogawa $\mathrm{T}$ et al. Intravenous immunoglobulin therapy for diabetic amyotrophy. Intern Med. 2001;40:349-52.

16. O'Neill BJ et al. Treatable lumbosacral polyradiculitis masquerading as diabetic amyotrophy. J Neurol Sci. 1997;151(2):223-5.

17. Smith AG, Singleton JR. Diabetic neuropathy. Continuum: lifelong learning in neurology. 2012;18(1):6084.

18. Tamburin S, Zanette G. Intravenous immunoglobulin for the treatment of diabetic lumbosacral radiculoplexus neuropathy. Pain Med. 2009;10(8):1476-80.

19. Tamburin $\mathrm{S}$ et al. Long-term response of neuropathic pain to intravenous immunoglobulin in relapsing diabetic lumbosacral radiculoplexus neuropathy. A case report. Pain Practice. 2014;14(2):E85-90.

20. Taylor BV, Dunne JW. Diabetic amyotrophy progressing to severe quadriparesis. Muscle Nerve. 2004;30(4):505-9

21. Tracy JA et al. Microvasculitis in diabetic lumbosacral radiculoplexus neuropathy. J Clin Neuromuscul Dis. 2009;11(1):44-8.

22. Younger DS. Diabetic lumbosacral radiculoplexus neuropathy: a postmortem studied patient and review of the literature. J Neurol. 2011;258(7):1364-7.

23. Barohn RJ et al. The Bruns-Garland syndrome (diabetic amyotrophy). Revisited 100 years later. Arch Neurol. 1991;48(11):1130-5.

24. Casey EB, Harrison MJ. Diabetic 
amyotrophy: a follow-up study. $\mathrm{Br}$ Med J. 1972;1(5801):656-9.

25. Dyck PJ et al. Microvasculitis and ischemia in diabetic lumbosacral radiculoplexus neuropathy. Neurology. 1999;53(9):2113-21.

26. Hamilton CRJ et al. Diabetic amyotrophy: clinical and electronmicroscopic studies in six patients. Am J Med Sci. 1968;256(2):81-90.

27. Leedman PJ et al. Diabetic amyotrophy: reassessment of the clinical spectrum. Aust N Z J Med. 1988;18(6):768-73.

28. Llewelyn JG et al. Epineurial microvasculitis in proximal diabetic neuropathy. J Neurol. 1998;245(3):159-65

29. Said $G$ et al. Nerve biopsy findings in different patterns of proximal diabetic neuropathy. Ann Neurol. 1994;35(5):559-69.

30. Dyck PJ et al. Microvasculitis in nondiabetic lumbosacral radiculoplexus neuropathy (LSRPN): similarity to the diabetic variety. J Neuropathol Exp Neurol. 2000;59(6):525-38.

31. Kawamura $\mathrm{N}$ et al. Inflammatory mediators in diabetic and nondiabetic lumbosacral radiculoplexus neuropathy. Acta Neuropathol. 2008;115(2):231-9.

32. Dyck PJB et al. The multicenter, double-blind controlled trial of IV methylprednisolone in diabetic lumbosacral radiculoplexus neuropathy. Neurology. 2006;65(5 Suppl 2):A191.

33. Krendel DA et al. Successful treatment of neuropathies in patients with diabetes mellitus. Arch Neurol. 1995;52(11):1053-61.

34. Pascoe MK et al. Subacute diabetic proximal neuropathy. Mayo Clin Proc. 1997;72(12):1123-32.

35. Jaradeh SS et al. Progressive polyradiculoneuropathy in diabetes: correlation of variables and clinical outcome after immunotherapy.
J Neurol Neurosurg Psychiatry. 1999;67(5):607-12.

36. Chan YC et al. Immunotherapy for diabetic amyotrophy. Cochrane Database Syst Rev. 2017(7):CD006521.

37. Tesfaye S et al. Painful diabetic peripheral neuropathy: consensus recommendations on diagnosis, assessment and management. Diabetes Metab Res Rev. 2011;27(7):629-38.

38. Sinnreich $M$ et al. Diabetic neuropathies. Classification, clinical features, and pathophysiological basis. Neurologist. 2005;11(2):63-79.

39. Coppack SW, Watkins PJ. The natural history of diabetic femoral neuropathy. Q J Med. 1991;79(288):307-13.

40. Dyck PJ et al. Non-diabetic lumbosacral radiculoplexus neuropathy: natural history, outcome and comparison with the diabetic variety. Brain. 2001;124(Pt 6):1197-207. 